\title{
Factores nutricionales y rendimiento académico Estudio piloto
}

\author{
Alberto Gimeno Tena \\ al362112@uji.es \\ Aurora Esteve Clavero \\ estevea@uji.es
}

\section{Resumen}

Introducción: Los universitarios son un grupo poblacional susceptible a los malos hábitos nutricionales. Estudios sugieren que existe relación entre hábitos de vida saludables y rendimiento académico. Mediante acciones de educación para la salud se pueden mejorar hábitos de vida, con el objetivo de contribuir a mejorar resultados académicos.

Metodología: Estudio piloto observacional transversal. Muestra poblacional de 20 estudiantes elegidos por muestreo por conveniencia. Recogida de datos mediante cuestionario diseñado ad hoc, revisado por un comité de expertos. Se realiza análisis descriptivo preliminar. Para determinar si hay asociación entre variables se realiza la prueba de chi-cuadrado.

Resultados: El $80 \%$ de los estudiantes presenta una adherencia media a la dieta mediterránea y el $20 \%$, una adherencia baja. Un $65 \%$ presenta un rendimiento académico por encima de la media. Las mujeres presentan en mayor proporción que los hombres una nota por encima de la media $(83 \%)$ $(p<0,05)$. El $77 \%$ de los estudiantes que realizan las 5 comidas diarias tienen un rendimiento académico por encima de la media $(p<0,05)$.

Discusión y conclusiones: Ninguno de los estudiantes tiene adherencia alta a la dieta mediterránea, esto hace aconsejable una intervención para mejorar los hábitos de alimentación. Existe asociación positiva entre buenos hábitos de alimentación y nota media del expediente; por tanto, promover estos hábitos puede contribuir a la mejora del rendimiento académico.

Palabras clave: nutrición, rendimiento académico, dieta mediterránea, estilo de vida saludable, estudiantes.

\section{Abstract}

Introduction: University students are a population group susceptible to bad nutritional habits. Studies suggest that there is a relationship between healthy lifestyles and academic performance. Through health education actions, life habits can be improved, with the aim of improving academic results. 
Method: Observational cross-sectional pilot study. Population sample: 20 students chosen by convenience sampling. Data collection by questionnaire designed ad hoc, reviewed by a professional committee. A preliminary descriptive analysis is carried out. To determine if there is an association between variables, the chi-square test is performed

Results: $80 \%$ present an average adherence to the Mediterranean diet; and $20 \%$ a low adhesion. $65 \%$ present an academic performance above the average. Women present a greater proportion than men a note above the average $(83 \%)(p<0.05) .77 \%$ of the students who perform the 5 daily meals have an academic performance above the average $(p<0.05)$.

Discussion and conclusion: none of the students has high adherence to the Mediterranean diet, this advises an intervention to improve nutritional habits. There is a positive association between good eating habits and the average grade of the academic record, therefore promoting these habits can contribute to the improvement of academic performance.

Keywords: nutrition, academic performance, Mediterranean diet, healthy lifestyle, students.

\section{Introducción}

Los estudiantes universitarios se consideran susceptibles de adquirir hábitos nutricionales poco saludables, debido principalmente a encontrarse en situaciones de constante cambio, como son largas horas de estudio y clases con horarios diversos que no les permiten seguir una rutina en referencia a los hábitos de vida, al aumento de la vida nocturna y también a la escasez económica. Este grupo poblacional realiza en gran medida acciones que suponen ayunos prolongados, omiten comidas de forma reiterativa debido a los factores comentados anteriormente y, además, consumen en gran proporción alimentos ricos en grasas y azúcares (Cumillaf et al. 2015)

Existen diversos estudios que afirman que tanto el adecuado peso corporal, en concreto el índice de masas corporal (IMC), como el correcto estado nutricional se ha asociado positivamente al rendimiento académico y a la nota media del expediente académico del estudiante. Existe una creciente evidencia que asocia la obesidad y la actividad cognitiva, incluyendo la función ejecutiva, la atención y la memoria (Cumillaf et al. 2015; Stea y Torstveit 2014).

Varias investigaciones afirman que tener unos hábitos nutricionales donde predominan los alimentos saludables, además de realizar las 5 comidas diarias recomendadas, se asocia, todo ello, a una menor probabilidad de problemas de aprendizaje en estudiantes. Por tanto, llevar una dieta de elevada calidad nutricional y alimenticia mejora el rendimiento académico (Stea y Torstveit 2014; Florence, Asbridge y Veugelers 2008).

Mediante un estudio a estudiantes españoles se afirma que una combinación de comportamientos de salud, en concreto hábitos nutricionales, puede tener una influencia positiva en el rendimiento académico de las adolescentes (Martínez-Gómez et al. 2012).

En relación con la dieta mediterránea y la adherencia a esta, existen estudios que afirman que su correcta adherencia, midiéndola mediante el cuestionario 
Mediterranean Diet Adherence Screener (MEDAS), influye positivamente en la cognición, en comparación con una dieta baja en grasas. La dieta mediterránea se caracteriza por el uso del aceite de oliva como la principal grasa culinaria y el alto consumo de alimentos de origen vegetal (fruta fresca como principal postre diario, frutos secos, verduras, legumbres y cereales mínimamente procesados). También incluye un consumo de moderado a alto de pescados y mariscos, un bajo consumo de mantequilla u otros productos lácteos y productos cárnicos, un consumo de alimentos frescos poco procesados y de temporada, y un consumo habitual y regular, pero moderado, de alcohol, preferentemente de vino tinto durante las comidas (Hernández Martínez-Lapiscina et al. 2013; Fundación dieta mediterránea s. f.).

Otros hábitos nutricionales, como realizar el desayuno de forma constante y realizarlo además de un modo completo, incluyendo en el mismo desayuno un alimento, al menos, del grupo de lácteos, cereales y fruta, se han relacionado con el rendimiento académico. Por tanto, al identificar estos comportamientos y establecer intervenciones de educación para la salud se pueden modificar los logros académicos, hallazgos que justifican intervenciones e iniciativas en el ámbito de la educación y la promoción de la salud. Estos aspectos comentados están involucrados en primera persona con el personal de enfermería y sus funciones y aportaciones a la población y a los usuarios sanitarios (Faught, Gleddie, Storey, Davison y Veugelers 2017; Fernández Morales, Aguilar Vilas, Mateos Vega y Martínez Para 2008).

En este sentido, los objetivos del presente estudio son analizar los factores nutricionales y el rendimiento académico de los estudiantes de la Universitat Jaume I $y$, además, determinar si hay asociación entre las variables utilizadas en el estudio en el contexto de estos estudiantes universitarios. Este estudio puede ser relevante al identificar los factores susceptibles de modificación mediante acciones de educación para la salud, marco en el que la enfermería tiene un papel importante.

\section{Metodología}

Se trata de un estudio piloto observacional transversal, mediante técnica de encuesta, realizado durante los meses de octubre de 2018 a abril de 2019, en la Universitat Jaume I de Castellón, España.

La población de estudio son los estudiantes de la Universitat Jaume I. Los criterios de inclusión son: estudiantes de ambos sexos y estudiantes que brinden su consentimiento para participar en la investigación. Los criterios de exclusión son: estudiantes que padezcan alguna enfermedad crónica que requiera una dieta específica.

La muestra estuvo formada por 20 estudiantes que cumplían los criterios de inclusión de la Universitat Jaume I y fueron seleccionados por muestreo por convenien cia.

Las variables del estudio son las siguientes:

- Edad.

- Género.

- Rendimiento académico, mediante la nota media del expediente académico.

- Factores nutricionales:

- Índice de masas corporal (IMC).

- Adherencia a la dieta mediterránea, mediante el cuestionario Mediterranean Diet Adherence Screener (MEDAS). 
- Desayuno todas las mañanas de forma constante; o no, en contraposición.

- Desayuno completo (el mismo desayuno contiene fruta, lácteos y cereales); o no, en contraposición.

- Número de comidas que realizan al día.

- Lugar donde realizan la mayor parte de las comidas.

El cuestionario MEDAS mide la adherencia a la dieta mediterránea mediante la valoración de 14 puntos. Un artículo evaluó su validez relativa y constructiva para el estudio de la prevención con dieta mediterránea (PREDIMED) $\mathrm{y}$, además, es ampliamente utilizado en estudios relacionados con hábitos nutricionales de la dieta mediterránea (Schröder et al. 2011; Hernández Martínez-Lapiscina et al. 2013).

A los estudiantes que cumplieron los criterios de inclusión se les facilitó un cuestionario autoadministrado, diseñado ad hoc mediante Google Forms. Dicho cuestionario incluye las variables anteriormente comentadas. El cuestionario fue revisado por una mesa de expertos que incluía tres profesionales enfermeros profesores y otro psicólogo, de la misma Universitat Jaume I.

En referencia al análisis estadístico, la explotación de la base de datos se realizó con el paquete R-Commander. Se realizó una primera parte descriptiva para todas las variables recogidas y, en una segunda fase, se realizó un contraste de hipótesis mediante el test chi-cuadrado para contrastar variables categóricas y determinar si existía relación entre las diferentes variables y el rendimiento académico. Se consideraron asociaciones con significación estadística con un valor de $p<0,05$.

\section{Resultados}

En el estudio se incluyeron 20 estudiantes con una edad media de 20,45 años. De primer curso de los diferentes grados de la universidad, el $60 \%$ de estudiantes son hombres y el $40 \%$ son mujeres. De segundo, el $100 \%$ son hombres. De tercer curso, el $75 \%$ de estudiantes son mujeres y el $25 \%$ son hombres. Y de cuarto curso, el $100 \%$ son mujeres.

Un $65 \%$ de los estudiantes presenta un rendimiento académico por encima de la media, siendo la nota media de la muestra poblacional un 7,2 sobre 10.

El $75 \%$ de los estudiantes tienen un peso normal (IMC entre 18,5 y 24,9 ) y el $25 \%$ restante tiene sobrepeso (IMC entre 25 y 29,9). El $80 \%$ de los estudiantes presentan una puntuación en el cuestionario Predimed entre 6 y 11, lo que significa que su adherencia a la dieta mediterránea es media. El $20 \%$ presenta una puntuación menor que 6 , es decir, una adherencia baja a la dieta mediterránea. Ninguno de estos presenta una adherencia alta a la dieta mediterránea. El $70 \%$ de los estudiantes realizan el desayuno todas las mañanas de un modo regular y constante, mientras que el $30 \%$ de los estudiantes no realizan el desayuno o lo realizan de vez en cuando. El $65 \%$ de los estudiantes realiza el desayuno de forma completa incluyendo hidratos de carbono, lácteos y fruta; en contraposición, el $35 \%$ realiza un desayuno incompleto. El $20 \%$ de los estudiantes realiza 3 comidas diarias, el $15 \%$ realiza 4 comidas diarias y el $65 \%$ de los estudiantes realiza las 5 comidas recomendadas diarias. El $70 \%$ de los estudiantes realiza la mayor parte de las comidas principales (comida y cena) en casa; en cambio, el $30 \%$ restante las realiza en la universidad. El $73 \%$ de los estudiantes con peso normal tienen un rendimiento académico por encima de la media. Al igual que el $69 \%$ de los estudiantes que muestran una adherencia media a la dieta mediterránea. 
A continuación, en la tabla 1, se presentan los resultados obtenidos del cuestionario.

Tabla 1

Rendimiento académico en función de las variables del estudio

\begin{tabular}{|c|c|c|c|c|c|}
\hline & \multicolumn{4}{|c|}{ Rendimiento académico } \\
\hline & & $\begin{array}{l}\text { Por encima } \\
\text { de la media }\end{array}$ & $\begin{array}{l}\text { Por debajo } \\
\text { de la media }\end{array}$ & $\mathrm{X}^{2}$ & p-valor* \\
\hline \multirow{2}{*}{ IMC } & Peso normal & $11(73 \%)$ & $4(27 \%)$ & \multirow{2}{*}{1,83} & \multirow{2}{*}{0,176} \\
\hline & Sobrepeso & $2(40 \%)$ & $3(60 \%)$ & & \\
\hline \multirow{2}{*}{ Género } & Hombre & $3(37,5 \%)$ & $5(62,5 \%)$ & \multirow{2}{*}{4,43} & \multirow{2}{*}{0,0352} \\
\hline & Mujer & $10(83 \%)$ & $2(17 \%)$ & & \\
\hline \multirow{2}{*}{$\begin{array}{l}\text { Adherencia } \\
\text { dieta } \\
\text { mediterránea }\end{array}$} & Media & $11(69 \%)$ & $5(31 \%)$ & \multirow{2}{*}{0,49} & \multirow{2}{*}{0,4819} \\
\hline & Baja & $2(50 \%)$ & $2(50 \%)$ & & \\
\hline \multirow{2}{*}{$\begin{array}{l}\text { Desayuno } \\
\text { todos los días }\end{array}$} & Sí & $10(71 \%)$ & $4(29 \%)$ & \multirow{2}{*}{0,84} & \multirow{2}{*}{0,3572} \\
\hline & No & $3(50 \%)$ & $3(50 \%)$ & & \\
\hline \multirow{2}{*}{$\begin{array}{l}\text { Desayuno } \\
\text { completo }\end{array}$} & Sí & $3(43 \%)$ & $4(57 \%)$ & \multirow{2}{*}{2,32} & \multirow{2}{*}{0,1276} \\
\hline & No & $10(77 \%)$ & $3(23 \%)$ & & \\
\hline \multirow{3}{*}{$\begin{array}{l}\text { Comidas } \\
\text { diarias }\end{array}$} & 3 comidas & $3(75 \%)$ & $1(25 \%)$ & \multirow{3}{*}{6,56} & \multirow{3}{*}{0,0377} \\
\hline & 4 comidas & - & $3(100 \%)$ & & \\
\hline & 5 comidas & $10(77 \%)$ & $3(23 \%)$ & & \\
\hline \multirow{2}{*}{$\begin{array}{l}\text { Lugar donde } \\
\text { suele comer }\end{array}$} & Casa & $9(64 \%)$ & $5(36 \%)$ & \multirow{2}{*}{2,35} & \multirow{2}{*}{0,9185} \\
\hline & Universidad & $4(67 \%)$ & $2(33 \%)$ & & \\
\hline
\end{tabular}

*Se considera significación estadística si p-valor $<0,05$

Se ha relacionado la variable de rendimiento académico con las otras distintas variables.

No se ha logrado establecer una asociación estadísticamente significativa entre el rendimiento académico y la adherencia a la dieta mediterránea. En referencia al desayuno tampoco se ha encontrado asociación con el rendimiento académico (ni con si los estudiantes desayunan todos lo días, ni con si realizan un desayuno completo). El lugar donde suelen realizar los estudiantes las principales comidas tampoco tiene asociación con el rendimiento académico.

Como se puede observar en la figura 1, las mujeres presentan, en mayor proporción que los hombres $(37,5 \%)$, una nota por encima de la media $(83 \%)(p<0,05)$. 


\section{Rendimiento académico / Género}

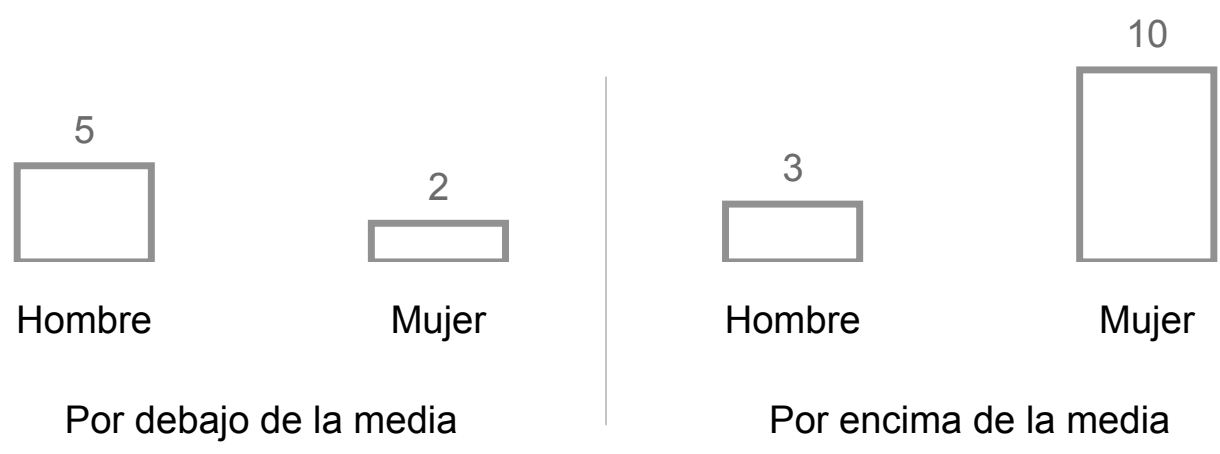

Figura 1. Relación entre rendimiento académico y género.

En relación con el número de comidas diarias que realizan los estudiantes, el $77 \%$ de los estudiantes que realizan las 5 comidas diarias tienen un rendimiento académico por encima de la media $(p<0,05)$. Obsérvese la figura 2.

\section{Rendimiento académico / Número de comidas}

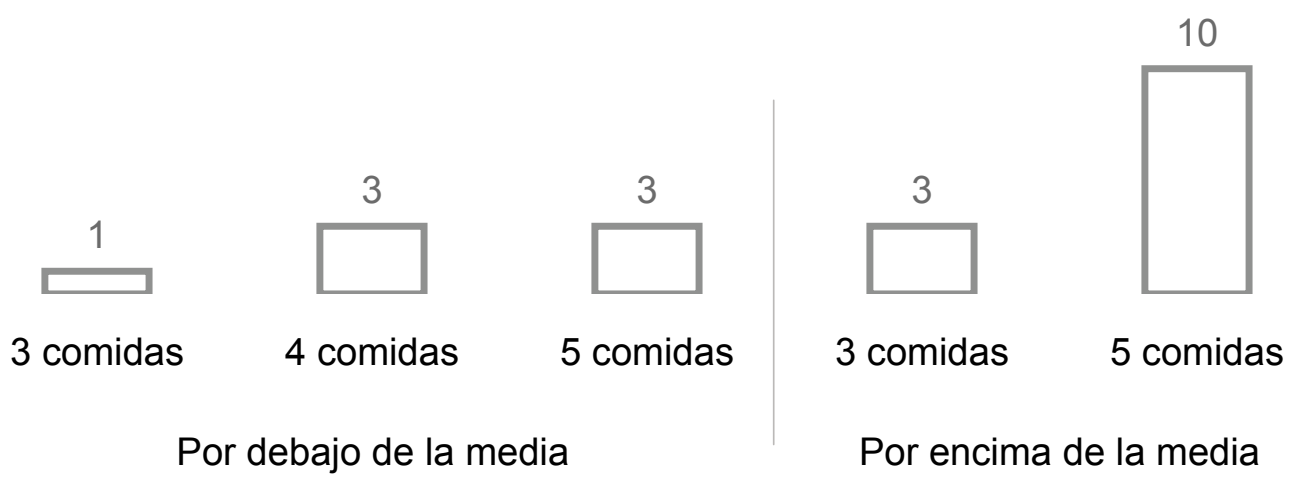

Figura 2. Relación entre rendimiento académico y número de comida diarias.

\section{Discusión y conclusiones}

El rendimiento académico en estudiantes universitarios puede verse influido por numerosos factores. Identificar factores nutricionales que influyan en él permitirá actuaciones encaminadas a reforzar o modificar hábitos que ayuden a mejorar el desempeño académico.

De los resultados anteriormente presentados, llama la atención que no se ha podido constatar una asociación estadísticamente significativa entre algunas de las variables nutricionales que tradicionalmente se han considerado relacionadas con el problema estudiado.

En el presente estudio no se encuentra relación entre el IMC y el rendimiento académico. En cambio, en el estudio de Logi Kristjánsson, Sigfúsdóttir y Allegrante (2010) afirma que el IMC sí está relacionado con la nota media del expediente académico de los estudiantes. Podría ser debido principalmente a que la muestra 
poblacional es muy reducida, según comentamos a continuación en las limitaciones del estudio. En cambio, sí que se ha observado que un gran porcentaje de alumnos $(25 \%)$ tiene sobrepeso; por tanto, intervenciones dietéticas y también relacionadas con la actividad física serían recomendadas para reducir esta tasa de sobrepeso en los alumnos de la universidad.

Según Hernández Martínez-Lapiscina et al. (2013), la adherencia a la dieta mediterránea está directamente relacionada con el rendimiento académico; no obstante, en nuestro estudio no se ha encontrado esta relación debido, en nuestra opinión, a la baja muestra poblacional como anteriormente hemos comentado. Independiente de la relación con el rendimiento, se observa que ninguno de los estudiantes tiene una adherencia alta a la dieta mediterránea, por lo que se aconseja una intervención, por ejemplo, por parte del personal de enfermería para mejorar y promover estos hábitos nutricionales relacionados con la dieta mediterránea de los estudiantes universitarios.

Si observamos las dos variables relacionadas con el desayuno (si los estudiantes lo toman o no de forma constante todos los días y si este se realiza de una forma completa, es decir, si toman en el mismo desayuno fruta, lácteos y cereales), observamos que hay disparidad entre nuestro estudio y el estudio de Fernández Morales, Aguilar Vilas, Mateos Vega y Martínez Para (2008) que afirma que el consumo de un desayuno poco completo e inadecuado puede influir en los resultados académicos y en la nota media de ciertas asignaturas en concreto. Esto podría ser debido a que la muestra poblacional de 20 estudiantes es muy reducida y el análisis probabilístico puede ser limitado.

Respecto al género de la población y su relación con el rendimiento académico, encontramos otro artículo de los autores Valladares et al. (2016) que afirma, al igual que en nuestro estudio, que la proporción de mujeres con mejor nota media del expediente es mayor que la de los hombres y que estas variables están relacionadas estadísticamente.

Por otro lado, se han encontrado similitudes entre los resultados de este estudio y los resultados de varios estudios (Faught, Gleddie, Storey, Davison y Veugelers 2017; Rehman, Zafar, Mohib, Hussain y Ali 2018; Stea y Torstveit 2014). El estudio de Faught, Gleddie, Storey, Davison y Veugelers (2017) afirma que mantener buenos hábitos nutricionales, como por ejemplo realizar las 5 comidas diaraias (desayuno, almuerzo, comida, merienda y cena) mejora el rendimiento académico de los estudiantes; al igual que el estudio de Rehman, Zafar, Mohib, Hussain y Ali (2018). En concreto, el estudio de Stea y Torstveit (2014) afirma que seguir un correcto patrón alimenticio y no saltarse ninguna de las 5 comidas diaras recomendadas, como muchos de los estudiantes que se encuentran en el periodo universitario, está positivamente relacionado con la nota media de los estudiantes.

Como limitaciones del presente estudio encontramos que se trata de un estudio piloto, por lo que el tamaño muestral es muy pequeño (20 estudiantes) y que, por tanto, el muestreo no es probabilístico, pero ha permitido valorar el cuestionario utilizado e identificar ciertos puntos de mejora.

A la vista de los resultados obtenidos puede concluirse que existe una asociación positiva entre los buenos hábitos de alimentación y la nota media del expediente académico.

Por lo tanto, al igual que con la adherencia a la dieta mediterránea y con el índice de masas corporal elevado, se aconseja uns intervención de educación para la salud, por 
ejemplo por parte del personal de enfermería, para poder promover hábitos de vida saludables y también nutricionales, con el objetivo secundario de poder mejorar el rendimiento académico y la nota media del expediente de los estudiantes.

\section{Referencias bibliográficas}

Cumillaf, Andrés Godoy, Custodio Fariña Herrera, Francisco Carcamo Mora, Bernice Medina Herrera, Elías Meneses Sandoval, Pablo Antonio Valdés Badilla, Relmu Gedda y Samuel Durán. 2015. «Asociación entre la condición física, estado nutricional y rendimiento académico en estudiantes de educación física». Nutrición Hospitalaria, 32(4): 1722-1728.

Faught, Erin L., Douglas Gleddie, Kate E. Storey, Colleen M. Davison y Paul J. Veugelers. 2017. «Healthy lifestyle behaviours are positively and independently associated with academic achievement: An analysis of self-reported data from a nationally representative sample of Canadian early adolescents». PLOS ON, 12(7): 1-14.

Fernández Morales, Irene, María Victorina Aguilar Vilas, Carmen José Mateos Vega y María Carmen Martínez Para. 2008. «Relación entre la calidad del desayuno y el rendimiento académico". Nutrición Hospitalaria, 23(4): 383-387.

Florence, Michel D., Mark Asbridge y Paul J. Veugelers. 2008. «Diet Quality and Academic Performance». Journal of School Health, 78(4): 209-215.

Fundación dieta mediterránea. s.f. «¿Qué es la dieta mediterránea?». https://dietamediterranea.com/nutricion-saludable-ejercicio-fisico/\#piramide

Hernández Martínez-Lapiscina, Elena, Pedro Clavero, Estefanía Toledo, Ramon Estruch, Jordi Salas-Salvadó, Beatriz San Julián, Ana Sánchez-Tainta, Emilio Ros, Cinta Valls-Pedret y Miguel Ángel Martínez-González. 2013. «Mediterranean diet improves cognition: the PREDIMED-NAVARRA randomised trial». Journal of Neurology, Neurosurgery \& Psychiatry, 84(12): 1318-1325.

Logi Kristjánsson, Álfgeir, Inga Dóra Sigfúsdóttir y John P. Allegrante. 2010. «Health Behavior and Academic Achievement Among Adolescents: The Relative Contribution of Dietary Habits, Physical Activity, Body Mass Index, and SelfEsteem». Health Education \& Behavior, 37(1): 51-64.

Martínez-Gómez, David, Oscar L. Veiga, Sonia Gómez Martínez, Belén Zapatera García, David Martínez-Hernández, María Elisa Calle y Ascensión Marcos. 2012. "Gender-specific influence of health behaviors on academic performance in Spanish adolescents: the AFINOS study». Nutricion Hospitalaria 27(3): 724730.

Rehman, Rehana, Amara Zafar, Aleena Mohib, Mehwish Hussain y Rabiya Ali. 2018. «Self-reported academic performance in relation to health behaviours among Bahria University students». Journal of the Pakistan Medical Association, 68(2): 195-199.

Schröder, Helmunt et al. 2011. «A Short Screener Is Valid for Assessing Mediterranean Diet Adherence among Older Spanish Men and Women». The Journal of Nutrition 141(6): 1140-1145.

Stea, Tonje H. y Monica K. Torstveit. 2014. «Association of lifestyle habits and academic achievement in Norwegian adolescents: a cross-sectional study». BMC Public Health, 14(1): 829.

Valladares, Macarena, Elizabeth Durán, Alexis Matheus, Samuel Durán, Ana María Obregon Rivas y R. Ramírez-Tagle. 2016. "Association between Eating Behavior and Academic Performance in University Students». Journal of the American College of Nutrition, 35(8): 699-703. 